\title{
Intrinsic Safety Evaluation for Urban Road Traffic Based on Information Entropy and Triangular Fuzzy Number
}

\author{
Gao Tingting ${ }^{1,2}$ Wang Wuhong ${ }^{1}$ and Xing Zhongyi ${ }^{1}$ \\ ${ }^{1}$ Beijing Institute of Technology, School of Mechanical Engineering, 100081, Beijing, China \\ ${ }^{2}$ Tian Jin University of Technology and education, School of Automobile and Transportation line, 300222, Tianjin, China
}

\begin{abstract}
Road traffic safety is a major problem, especially in low-and middle-income countries. This study attempted to evaluate traffic safety based on the concept of intrinsic safety, which can provide theoretical support for the road traffic management departments. The evaluation weight calculation about the pre-weight of the second level evaluation index based on the information entropy, and then calculates the final weight by combing qualitative and quantitative methods. The weight of the third level evaluation index using the triangular fuzzy number method. In order to verify the effectiveness and feasibility of the method via a case study. by computing the proportions of evaluation indexes based on the model, we found that the ranking is intrinsic human>intrinsic road and environment>intrinsic vehicle. However, the gap about the proportion of the three is small. Furthermore, the sub factors ranking influencing factors as follows: the human's alertness $=$ road and the environment's completeness $>$ vehicle's technical $>$ human's self-consciousness $>$ natural conditions $>$ vehicle's maintenance. Given the objectivity of the model in the paper, especially using the entropy weight method to calculate the weight of the index factors. The result of the evaluation for the model is similar with current situation of road traffic safety, thus, it can be taken as a valuable reference to promoting the traffic safety. So it can provide theoretical basis for urban road transport departments to formulate relevant policies.
\end{abstract}

\section{Introduction}

As the degree of motorization increase, improving traffic safety has become a main goal of transportation researchers across the world. Traditionally, traffic safety evaluations have relied mostly on crashes statistics as the main data source, and it is an important method to evaluate the traffic safety. To date, there are a large number of domestic and foreign experts or scholars who have been studying deeply on traffic safety, but they worked in most cases on their own specific road safety problems, to a large extent because the socioeconomic conditions, the motorization levels, and the road safety experiences are different from country to country and from region to region. By contrasting China and Japan in the Asian countries in 2012, it can be found that the death toll from traffic accidents was respectively 59997 and 4411 , the mortality rate per ten thousand cars was $2.5 \%$ and $0.58 \%$, and the mortality rate per 100000 people was $4.45 \%$ and $3.45 \%$. Therefore, it is necessary to evaluate the road traffic safety. To date, there are a large number of domestic and foreign experts or scholars who have been studying extensively on traffic safety: R - J • Smeed in University of London established a Smeed model about ownership of motor vehicles based on population and the number of road deaths in 1949; Solomon used vehicle speed to evaluate road traffic safety in 1964; Grazawa established an urban Traffic Safety Evaluation System with a relatively wide layered structure. Jake Kononov and Bryan Allery proposed SPFs (Safety Performance Functions), and subdivided the road traffic safety level into Loss I , Loss II , Loss III, Loss IV four levels. Road traffic safety evaluation of China was relatively new, Liu Shiqi built a highway safety evaluation model Lu in 2003 and Zhang in 2004 evaluated the study transportation system from the perspective of its security; Shao established a road traffic safety evaluation system more comprehensively in 2004 . The paper combined with information entropy to determine the index weight evaluation method based on triangular fuzzy number, and applies it to the nature of traffic safety evaluation, reduce the interference of subjective factors too much, Different from the traditional the third indexes, which focus on the characteristics of the second indexes, the number of the third indexes in the study is a few but its scope is generally fairly comprehensive. the essence of improving road safety evaluation index that affect the ranking on the level of road traffic safety and provide technical support and theoretical support for the positive [1]-[6]. 


\section{Road intrinsic safety evaluation index}

Evaluation indexes are the key of the evaluation model, and the premise of obtaining the real objective results. To choose the evaluation indexes correctly, the urban traffic system has to be analyzed comprehensively. The selected indexes should be able to reflect the impaction of various factors maximal degree, and the selected indexes should be able to determine the safety of transportation system maximal degree intrinsic.

Road traffic system is composed of human, vehicles, roads and the environment. Any one factor's disorder of transportation system can lead to accidents. Therefore, the indexes should meet the following principles: comprehensiveness and level, initiative and passive, and static and dynamic. Evaluation based on intrinsic safety by questionnaires shown in Fig. 2. The evaluation system of road traffic safety as the target layer, the human, vehicle and road-layer model as the rule layer, the consciousness and alertness of human, the technical and maintenance of vehicles, the completeness and natural condition of roads and environment as decision layer.

\subsection{Human}

Human is an active factor that has a great impact on road traffic safety. Whether drivers or ride personnel comply with traffic regulations and, have high safety consciousness constitute consciousness evaluation index; whether the driver's operation is skilled, and whether there are errors constitute alertness evaluation index.

\subsection{Vehicle}

Vehicle is a passive factor that also affects road traffic safety. Human - vehicle - environment is a closed loop control system that has a feedback loop. The vehicle plays a major role in the feedback loop. Whether the vehicle's technical performance is in good condition, and the maintenance is proper directly determine whether the transportation system is safe or not, and therefore vehicle design based on intrinsic safety is an important aspect regarding road safety.

\subsection{Road \& environment}

Roads \& environment is a subsystem of the transportation system as about the people - vehicle - environment, which is a place to accommodate people and vehicles. It is a necessary condition for the protection of people and cars to work. Whether road marking and labeling are clear and whether signal control facilities are complete constitute the completeness index. In addition, the bad natural conditions and the difference between day and night have a direct impact on road traffic safety.

The evaluation system compared with the previous evaluation system, are based on road transport system of humane, vehicles and roads, but there are several advantages: Use either SI (MKS) or CGS as primary units. (SI units are encouraged.) English units may be used as secondary units (in parentheses). An exception would be the use of English units as identifiers in trade, such as "3.5-inch disk drive".

1) Though the three-grade index has a small number, but covers the whole;

2) The three-grade indexes are easy to calculate standardized evaluation matrix;

3) The three-grade indexes are based on intrinsic safety.

\section{Model}

The model is a method based on fuzzy mathematics theory, and it is a more practical approach, and it can evaluate for the influence factors on multivariate fuzzy evaluation, especially for harder to quantify, and have strong fuzzy problem.

\subsection{Standard matrix}

General the dimension and magnitude of the model will be divided into two categories, one is the bigger the more excellent, and the other is the smaller the more excellent. Corresponding to these two types of matrix are shown as formula (1) and formula (2): [7]-[9]

The bigger the more excellent:

$$
r_{i j}=\frac{x_{i j}-\min \left(x_{j}\right)}{\max \left(x_{j}\right)-\min \left(x_{j}\right)}
$$

The smaller the more excellent:

$$
r_{i j}=\frac{\max \left(x_{j}\right)-x_{i j}}{\max \left(x_{j}\right)-\min \left(x_{j}\right)}
$$

where: $x_{i j}-$ score of the $\mathrm{j}$ index about $\mathrm{i}$ index;

$\max \left(x_{j}\right)$ —

$\min \left(x_{j}\right)$ - the $\min$ of $\mathrm{j}$ column about score matrix.

\subsection{The weight of the index $w_{i}$}

Determining the weight of the corresponding index is the key process of the evaluation, compared AHP method has a strong subjective to entropy method, it can better reflect the evaluation index weights. Weight refers to the standard is as follow formula (3) and (4): [10]-[11].

$$
\begin{gathered}
H_{j}=-\frac{\sum_{i=1}^{m} f_{l j} \ln f_{i j}}{\ln m}(i=1,2, \ldots, m ; j=1,2, \ldots n) \\
f_{i j}=r_{i j} / \sum_{i=1}^{m} r_{i j} \quad w_{i}=\frac{\left(1-H_{j}\right)}{\left(n-\sum_{i=1}^{n} H_{j}\right)}
\end{gathered}
$$

where: $r_{i j}$ - - judgment matrix after specification;

$m$ - - the number of rows of the matrix;

$n$ - - the number of columns of the matrix

\subsection{Triangular fuzzy number model}


Triangular fuzzy number is a special form of trapezoid fuzzy number, Fig. 1 is a function of the triangular fuzzy number, membership function is as shown in formula (5).

$$
f= \begin{cases}0 & x \in(-\infty, l] \\ \frac{x}{m-l}-\frac{l}{m-l} & x \in(l, m] \\ \frac{x}{m-n}-\frac{n}{m-n} & x \in(m, n] \\ 0 & x \in(n,+\infty)\end{cases}
$$

With respect to the addition, the multiplication and the scalar-multiplication of triangular fuzzy number in accordance with classical mathematical calculation rule, evaluation factors of triangular fuzzy number described more objective than traditional scoring assignment method. Triangular fuzzy number and scoring assignment respectively express road traffic safety evaluation indexes showed in Table 1 [12], [13].

Table 1. road traffic safety evaluation indexes based on triangular fuzzy number

\begin{tabular}{llllll}
\hline $\begin{array}{l}\text { road traffic } \\
\text { safety } \\
\text { evaluation level }\end{array}$ & very poor & Poor & ordinary & good & very good \\
\hline $\begin{array}{c}\text { fuzzy number } \\
\left(l^{\prime}, m^{\prime}, n^{\prime}\right)\end{array}$ & $\left(0,0, \frac{1}{4}\right)$ & $\left(0, \frac{1}{4}, \frac{1}{2}\right)$ & $\left(\frac{1}{4}, \frac{1}{2}, \frac{3}{4}\right)$ & $\left(\frac{1}{2}, \frac{3}{4}, 1\right)$ & $\left(\frac{3}{4}, 1,1\right)$ \\
\hline $\begin{array}{c}\text { score } \\
(\text { total point })\end{array}$ & {$[0 \sim 5.5)$} & {$[5.5 \sim 6.5)$} & {$[6.5 \sim 7.5)$} & {$[7.5 \sim 8.5)$} & {$[8.5 \sim 10]$} \\
\hline
\end{tabular}

Table 2. Tianjin city road traffic safety quantization indexes.

\begin{tabular}{|c|c|c|c|c|c|c|c|c|}
\hline & & $\begin{array}{l}\text { score } \\
\quad \text { (tota } \\
\text { l10poi } \\
\text { nt) }\end{array}$ & $r_{i j}$ & $W_{i}$ & $\begin{array}{l}\text { triangular } \\
\text { fuzzy } \\
\text { number }\end{array}$ & $\mathrm{p}_{i}$ & $\mathrm{I}\left(\mathrm{p}_{i}\right)$ & $w_{i}$ \\
\hline \multirow{2}{*}{ human } & Consciousness & 6.5 & 0 & \multirow{2}{*}{0.2589} & $\left(\frac{1}{4}, \frac{1}{2}, \frac{3}{4}\right)$ & $(0.0647,0.1295,0.1942)$ & 0.1295 & 0.16 \\
\hline & alertness & 7.8333 & 1 & & $\left(\frac{1}{2}, \frac{3}{4}, 1\right)$ & $(0.1295,0.1942,0.2589)$ & 0.1942 & 0.24 \\
\hline \multirow{2}{*}{ vehicle } & technical & 7.5 & $\begin{array}{l}0.85 \\
71\end{array}$ & \multirow{2}{*}{0.2248} & $\left(\frac{1}{2}, \frac{3}{4}, 1\right)$ & $(0.1124,0.1686,0.2248)$ & 0.1686 & 0.21 \\
\hline & Maintenance & 5.8333 & $\begin{array}{l}0.14 \\
28 \\
\end{array}$ & & $\left(0, \frac{1}{4}, \frac{1}{2}\right)$ & $(0,0.0562,0.1124)$ & 0.0562 & 0.07 \\
\hline \multirow{2}{*}{$\begin{array}{l}\text { road \& } \\
\text { environme } \\
\text { nt }\end{array}$} & Completeness & 7.6667 & 1 & \multirow{2}{*}{0.2589} & $\left(\frac{1}{2}, \frac{3}{4}, 1\right)$ & $(0.1295,0.1942,0.2589)$ & 0.1942 & 0.24 \\
\hline & $\begin{array}{l}\text { natural } \\
\text { condition }\end{array}$ & 5.5 & 0 & & $\left(0, \frac{1}{4}, \frac{1}{2}\right)$ & $(0,0.0647,0.1295)$ & 0.0647 & 0.08 \\
\hline
\end{tabular}

Index weight $W_{i}$ were calculated by the entropy weight, and multiplied by the fuzzy number for each index, obtained the fuzzy number of the pre-sorted by the expected formula

$$
\mathrm{I}\left(\mathrm{p}_{i}\right)=\frac{\mathrm{W}_{i} \cdot\left(\mathrm{l}^{\prime}+2 \mathrm{~m}^{\prime}+\mathrm{n}^{\prime}\right)}{4}
$$

Final weight of each evaluation factors $w_{i}$, can be sorted

$$
w_{i}=\frac{\mathrm{I}(\mathrm{p})}{\sum \mathrm{I}(\mathrm{p})}
$$

\section{Case study}

Take Tianjin as an example, we study on the impact of road traffic safety level of the essence of man, nature and he nature of road vehicles and the environment by the use of a questionnaire, we distributed 100 questionnaires were returned 95 valid questionnaires, obtained the road intrinsic safety score matrix A as follows:

$$
A=\left(\begin{array}{ll}
6.5 & 7.8333 \\
7.5 & 5.8333 \\
7.6667 & 1
\end{array}\right)
$$

And obtained standard matrix by The bigger the more excellent:

$$
R=\left(\begin{array}{ll}
0 & 1 \\
0.8571 & 0.1428 \\
1 & 0
\end{array}\right)
$$

We obtained the data by using equation (3) - (6) as shown in Table 2.

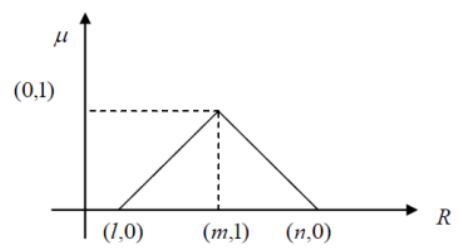

Figure 1. Function of the triangular fuzzy number. 
The results show that, the intrinsic factors of urban road traffic safety, the human factor occupies $40 \%$, the road and environment holds $32 \%$, the vehicle holds $28 \%$, the index order of factors: intrinsic human> intrinsic road and Environment> intrinsic vehicle, However, the difference of the proportion is little; and the proportion of sub-factors: human's alertness and completeness of the roads and environment are equally important, occupy $24 \%$, the vehicle technical occupies $21 \%$, the human consciousness occupies $16 \%$, natural conditions occupies $8 \%$, vehicle maintenance occupies $7 \%$.the order of subfactors: human's alertness $=$ road and environment completeness $>$ vehicle technical $>$ human consciousness $>$ natural conditions $>$ vehicle maintenance .

\section{Conclusion}

The main purpose of the paper is to analyze the evaluation indexes of impacting urban road traffic intrinsic safety, the paper calculated the index weight by using the information entropy, more objective than the conventional AHP method, to sort based on triangular fuzzy number method, more comprehensive than in the past, the calculated result may provide the basis for urban road transport sector. The main conclusions are as follows:

1) model was the impact of urban road traffic safety is the most essential factor in who holds $40 \%$ share, and the city that reason about $80 \%$ of road accidents are caused by humans is consistent;

2) The human's alertness and completeness of roads are the two most important factors that affect the intrinsic road traffic safety, occupy $24 \%$, according to statistics, traffic accidents, especially in largescale accidents, mainly due to the drivers' misuse, unresponsive and road transport facilities incomplete.

There are some limitations in the paper; the evaluation assignment was using the traditional questionnaire method, so questionnaire setting and its coverage problem will affect the value of the index, so this is a serious problem next paper.

\section{Acknowledgment}

This research has been financed by The National Natural Science Fund (NO.51408417), Tianjin University Science and Technology Development Fund (NO.20140917)

\section{References}

1. Andreessen D. Linking Deaths with Vehicles and Population [J]. Traffic Engineering and control, , 26;547-549, Nov. (1985)

2. Nain, Rental. Road Safety Model Some Fundamental Ideas: Transportation Research Record, National Research Council, Washington,D.C,1963,48-57

3. Ghazwan, al-Haji. Road Safety Development Index(RSDI)[D]. Sweden: Linkoping University, (2003)

4. Jake Kononov, Bryan K.Alley. Explicit Consideration of safety in transportation planning and project scooping[C]. Transportation Research Board 2004 Annual Meeting, 10:201-223, (2004)

5. Shao Zufeng. comprehensive evaluation of city road traffic safety[J]. Scholar Research, (2004): 22-24

6. Yu Rende, Shi Peng. The research on road traffic security evaluation based on the theory of fuzzy[J].Mathematics in Practice and Theory, 38 (7):109-116, (2008)

7. Chen Hong, Zhou Jibiao. Safety evaluation indexes and method for traffic environment of highway tunnels [J].Journal of Chang'an University, 33(4):5461, (2013).

8. Zheng Rui,Yang Zhenhong. Research on fuzzy comprehensive evaluation system of tailings based on entropy technology [J]. Journal of Science and Technology, 7(6):107-111, (2011)

9. Zhou Wei, Li Xiaojing. A comprehensive evaluation method based on information entropy [J]. Science Technology and Engineering, 10(23): 5839-5843, 2010

10. Qin Chen, Chen Xiaofang. Reaearch on safety Three-level evaluation of tailings reservoir based on FAHP-IE method [J]. Control Engineerin of China, 21(6): 995-1000, (2014)

11. Liu Honghong, Wang Ni. Fuzzy assessment of city ecosystem health based on entropy weight method [J]. Engineering Journal of Wuhan University, 47(6):755-759, (2014)

12. Metin Dadeviren. Developing a fuzzy analytic hierarchy process(AHP) model for behavior-based safety management[J]. Information Science, (178):1717-1733, (2008)

13. Si Shubin, Sun Shudong. A Weighted Analysis of an Integrated Logistics Support Based on Triangular Fuzzy Number [J]. Journal of Northwestern Polytechnical University, 22(6):689-694, (2004) 